\title{
Magnetospheric electron density model inferred from Polar plasma wave data
}

\author{
R. E. Denton, ${ }^{1}$ J. Goldstein, ${ }^{2}$ J. D. Menietti, ${ }^{3}$ and S. L. Young ${ }^{1,4}$ \\ Received 18 October 2001; revised 28 March 2002; accepted 14 May 2002; published 20 November 2002.
}

[1] Observations of the electron density $n_{\mathrm{e}}$ based on measurement of the upper hybrid resonance frequency by the Polar spacecraft Plasma Wave Instrument (PWI) are available for March 1996 to September 1997, during which time the Polar orbit sampled all magnetic local time (MLT) values three times. Using the entire data set, we bin the $n_{\mathrm{e}}$ values with respect to maximum field line radius $L R_{\mathrm{E}}$ and radius $R$ to generate an average density model for $3.5 \leq L \leq 7.8$ and $3 R_{\mathrm{E}} \leq R \leq L R_{\mathrm{E}}$. While our method does not assume any particular functional form for the density dependence along field lines, we find that the dependence can be roughly described by the power law form $n_{\mathrm{e}}=n_{\mathrm{e} 0}\left(L R_{\mathrm{E}} / R\right)^{\alpha}$ with $\alpha$ having on average a value $0.2-0.9$ in the plasmasphere (inner $L$ region, $3.5 \leq L \leq 5.5$ ), and a value $1.6-2.1$ in the plasmatrough (outer $L$ region, $L \geq 7$ ). For instance, $\alpha=0.8 \pm$ 1.2 at $L=4.4$ and $\alpha=2.1 \pm 1.4$ at $L=7$ (where the errors noted are overestimates). This result is similar to a previous result based on the Polar PWI data but using a different method. Alternately, the scale length for field line variation of density $L_{\alpha} \equiv\left(L R_{\mathrm{E}}\right) / \sqrt{\alpha}=$ $5.5 \pm 4.1 R_{\mathrm{E}}$ across the entire region $4.4 \leq L \leq 7.8$ (where the error noted is an overestimate). The data is also separated according to MLT, and it is shown that in the dawn sector the density is depleted and the radial dependence is steeper (larger $\alpha$ ) than for other MLT sectors. A similar result holds when the averaged $K p$ value is large $(>2)$. INDEX TERMS: 2768 Magnetospheric Physics: Plasmasphere; 2730 Magnetospheric Physics: Magnetosphere_inner; 2731 Magnetospheric Physics: Magnetosphere—outer; 2753 Magnetospheric Physics: Numerical modeling; 7819 Space Plasma Physics: Experimental and mathematical techniques; KEYWORDS: electron density, field line dependence, plasmasphere

Citation: Denton, R. E., J. Goldstein, J. D. Menietti, and S. L. Young, Magnetospheric electron density model inferred from Polar plasma wave data, J. Geophys. Res., 107(A11), 1386, doi:10.1029/2001JA009136, 2002.

\section{Introduction}

[2] While the average properties of the equatorial plasma density in the magnetosphere have been at least approximately described [Carpenter and Anderson, 1992; Gallagher et al., 2000], the latitudinal density dependence along field lines is less well known. Methods used to infer the latitudinal density dependence along magnetic field lines include in situ spacecraft observations, passive remote sensing with whistler waves and ultra low frequency (ULF) toroidal Alfvén frequencies [see Goldstein et al., 2001, and references therein], and active remote sensing using radio waves [Reinisch et al., 2001]. Results from the DE-1 spacecraft indicate that the normal situation is for the electron density $n_{\mathrm{e}}$ to be fairly constant along field lines

\footnotetext{
${ }^{1}$ Department of Physics and Astronomy, Dartmouth College, Hanover, New Hampshire, USA.

${ }^{2}$ Department of Physics and Astronomy, Rice University, Houston, Texas, USA.

${ }^{3}$ Department of Physics and Astronomy, University of Iowa, Iowa City, Iowa, USA.

${ }^{4}$ Present address Air Force Research Laboratory, Hanscom Air Force Base, Massachusetts, USA.

Copyright 2002 by the American Geophysical Union. 0148-0227/02/2001JA009136\$09.00
}

both in the outer plasmasphere $(L \gtrsim 3)$ and in the plasmatrough (see discussion in Goldstein et al. [2001]). This result is consistent with the constant density predicted for a completely trapped population in a dipole field [Chan et al., 1994] and is also roughly consistent with a diffusive equilibrium solution (for example, Young et al. [1980]).

[3] In an earlier study, Angerami and Carpenter [1966] used ground-based whistler wave data to infer a field line density dependence consistent with diffusive equilibrium (nearly constant) in the plasmasphere but $n_{\mathrm{e}} \propto R^{-4}$ in the plasmatrough. This last result is the so-called "collisionless" dependence resulting from an exospheric equilibrium with no equatorially trapped particles (consistent with a Maxwellian at the top of the collisional region near the surface of the Earth) [Lemaire and Gringauz, 1998, and references therein]. However, Angerami and Carpenter's results depended on extrapolation of the radial dependence down to an altitude of $1000 \mathrm{~km}$ (altitude of the Alouette 1 satellite), a procedure which may be questioned (see discussion in Goldstein et al. [2001]).

[4] Another technique is to infer mass density $\rho_{\mathrm{m}}$ using toroidal (azimuthal) Alfvén wave frequencies (see references in Menk et al. [1999] and Denton et al. [2001]). Assuming a power law form for mass density $\rho=\rho_{\mathrm{eq}}\left(L R_{\mathrm{E}} / R\right)^{\alpha}$, Cummings et al. [1969] calculated toroidal eigenfrequencies at geo- 
synchronous orbit $(L=6.6)$. Takahashi and McPherron [1982], Engebretson et al. [1986], and Menk et al. [1999] matched ratios of these frequencies to observed frequencies to infer values of the equatorial mass density $\rho_{\mathrm{eq}}$ and values of a varying from 0-6. Price et al. [1999] and Denton et al. [2001] used a more general method to infer mass density without requiring the power law form.

[5] Very recently, radio imaging of the electron density has been accomplished using the Radio Plasma Image instrument on the IMAGE spacecraft [Reinisch et al., 2001]. Reinisch et al. describe the density variation in terms of a power law with respect to the magnitude of the magnetic field $B, n_{\mathrm{e}} \propto B^{\mathcal{K}}$. For the data set they analyzed, $\mathcal{K} \sim 1 / 4$ near the equator, $\sim 1$ at $\sim 40^{\circ}$ latitude, and $\sim 2$ at the highest latitudes. Making the approximation that the dipole field $B \sim$ $R^{-3}$, this translates to $n_{\mathrm{e}} \propto R^{-3 / 4}$ near the equator, $n_{\mathrm{e}} \propto R^{-3}$ at $40^{\circ}$ latitude, and $n_{\mathrm{e}} \propto R^{-6}$ at the highest latitudes (where, however, $B \sim R^{-3}$ may not be a good approximation).

[6] Goldstein et al. [2001] used observations of the electron density $n_{\mathrm{e}}$ based on measurement of the upper hybrid resonance frequency by the Polar spacecraft Plasma Wave Instrument (PWI) [Gurnett et al., 1995]. Assuming a power law form for the electron density

$$
n_{\mathrm{e}}=n_{\mathrm{e} 0}\left(\frac{L R_{\mathrm{E}}}{R}\right)^{\alpha},
$$

where $n_{\mathrm{e} 0}$ is the electron density at the equator, they used the fact that the Polar spacecraft orbit crossed particular $L$ shells at two different radial locations to infer values of $\alpha$. They found on average $\alpha=0.37 \pm 0.8$ for their plasmaspheric data set $\left(n_{\mathrm{e}} \geq 100 \mathrm{~cm}^{-3}\right)$ but $\alpha=1.7 \pm 1.1$ for their plasmatrough data set $\left(n_{\mathrm{e}}<100 \mathrm{~cm}^{-3}\right)$.

[7] For this paper we again use Polar PWI data, but this time we bin the data with respect to $L$ shell and radius $R$ to generate plots of the average electron density distribution as a function of $R /\left(L R_{\mathrm{E}}\right)$ without requiring the power law assumption (1). (We then use the power law description to model the results.) We define $L$ shell such that $L R_{\mathrm{E}}$ is the maximum radius to any point along a field line. For a dipole field, $L R_{\mathrm{E}}$ would be the equatorial radius; however, we will be using a nondipolar magnetic field model for which the maximum radius may be off-equator (nonzero magnetic latitude). Our data describe the magnetosphere in the region $3.5 \leq L \leq 7.8$ and $3 R_{\mathrm{E}} \leq R \leq L R_{\mathrm{E}}$. In some respects our model is rather crude. For instance, it averages at a particular $L$ value the data from both plasmasphere and plasmatrough (since the position of the plasmapause varies with time). Such an averaging procedure will clearly smear out the differences between these two conditions and may lead to a characterization which is not entirely accurate for either. Nevertheless, the radial distributions can be used as a point of reference for density modeling, and the information we find on the field line distribution provides perhaps the most complete description yet for the density dependence close to the equator. An outline of our paper is as follows: In section 2 we discuss the measurement of $n_{\mathrm{e}}$ and our data set. In section 3 we discuss our results, and we summarize our findings in section 4 .

\section{Data and Method}

[8] The electron density values used in this paper are obtained using the Polar Plasma Wave Instrument (PWI)

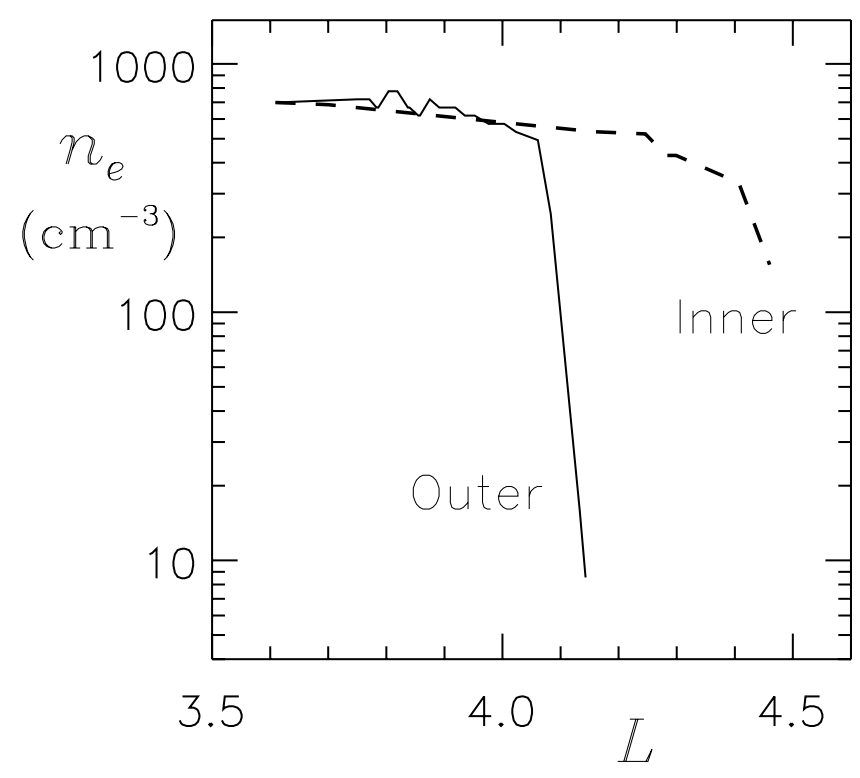

Figure 1. Electron density $n_{\mathrm{e}}$ as determined from the Polar PWI data measured on 1 April 1996, 0402-0508 UT versus $L$. The solid (dashed) curve represents the profile of $n_{\mathrm{e}}$ determined at the large (small) radius portion of the orbit.

[Gurnett et al., 1995]. The electron number density can be determined from noise emission which has an upper edge in frequency at the upper hybrid resonance (UHR) frequency [Goldstein et al., 2001]. This noise band is most commonly observed in the plasmasphere but can be detected in the plasmatrough.

[9] Figure 1 shows the electron density $n_{\mathrm{e}}$ as determined by the PWI on 1 April 1996, 0402-0508 UT. Owing to the nature of the Polar orbit [Goldstein et al., 2001] the trajectory of the spacecraft crosses $L$ shell values at two different radii. In Figure $1, n_{\mathrm{e}}$ is plotted versus $L$ for the outer (solid curve) and inner (dashed curve) portions of the orbit. Given a sharp drop-off in $n_{\mathrm{e}}$ which occurs at $L=4.1$ for the outer portion of the orbit but at $L=4.4$ for the inner portion, it is likely that both of these locations correspond to the plasmapause. The difference in $L$ value most likely corresponds to the fact that the measurements are taken at different local and universal time [Goldstein et al., 2001]. Note that there is a loss of the upper hybrid signal that prevents $n_{\mathrm{e}}<100 \mathrm{~cm}^{-3}$ from being measured on the inner portion of the orbit.

[10] The dropout of the signal mentioned in the last paragraph occurs because the intensity of the noise bands is often greatly reduced when Polar's trajectory is outside the plasmasphere and away from the equator, where the condition $f_{\mathrm{pe}} \gg f_{\mathrm{ce}}$ is often not satisfied. Considering the nature of the Polar spacecraft orbit during the period of our study $(1996,1997)$ (see Figure 1 of Goldstein et al. [2001]), this situation is most likely to occur when $L$ is large but the radius $R$ is small. The radial position of Polar is $R \sim 2 R_{\mathrm{E}}$ on the inner portion of the orbit where the upper hybrid signal drops out on 1 April 1996. A second cause for loss of potential density data occurs at the lowest $\mathrm{L}$ values, when Polar penetrates deepest into the high-density plasmasphere. In that case the preamplifiers located in the electric field antenna spheres often oscillate with a broad band signal 
centered near the UHR frequency which obscures the natural UHR signal [Kolesnikova et al., 2001]. Kolesnikova et al. report that this problem occurs primarily at $L<3$. Thus both of these problems occur when the radius to the spacecraft position is small. For this reason we have chosen to exclude data with $R<3 R_{\mathrm{E}}$ from the averages used to generate the density model.

[11] For this study, data were used from the entire time period for which the PWI was in operation, from 26 March 1996 to 16 September 1997, during which time the Polar spacecraft sampled all magnetic local time (MLT) values three times (the orbit rotates in local time owing to the Earth's revolution, and on each Polar orbit two opposite MLT values are sampled). Spectrogram images were scanned manually and data points were chosen by clicking with the mouse on the upper hybrid signature. For each data point a field line mapping program was used to map the spacecraft location to the position along the field line with maximum radius $R=R_{\max }$; we then define $L \equiv R_{\max } / R_{\mathrm{E}}$. In cases for which solar wind data were available $(92 \%$ of the data) we use the Tsyganenko 1996 [Tsyganenko, 1995] magnetic field model coupled with the International Geomagnetic Reference Field (IGRF) inner magnetic field model [Langel, 1991]. Where the solar wind data were not available we substitute the Tsyganenko 1989c model [Tsyganenko, 1989] for the outer field. Data were discarded for which the maximum radius along the field line and the radius corresponding to minimum magnetic field amplitude differed by more than $5 \%$. Our data set is $\sim 10$ times larger than that used by Goldstein et al. [2001]; altogether there are about 81,000 data points.

[12] We next generate a statistical model for the electron density in the magnetosphere for $3.5 \leq L \leq 7.8$. We have not separated data in the plasmasphere and plasmatrough, so this model represents the average density over these situations. Caution must be exercised in the interpretation of the plasmatrough densities. The PWI can in principal measure lower densities than those seen in Figure 1; the lowest density measured in our data set is $0.1 \mathrm{~cm}^{-3}$. However, as the outer trace (solid curve) in Figure 1 indicates, there can be a drop-off in signal in the plasmatrough even at large radius, causing us to miss measurements of low-density plasma (here with $n_{\mathrm{e}}<8 \mathrm{~cm}^{-3}$ ). Because of this, our statistical results for equatorial density may be an upper limit in the plasmatrough, especially when the averages yield a value $<10 \mathrm{~cm}^{-3}$.

\section{Results}

[13] Figure 2a shows the equatorial electron density $n_{\mathrm{e} 0}$ versus $L$ determined in $\Delta L=0.15$ bins from $L=3.3$ to 8.3 .

Figure 2. (opposite) Plot of (a) equatorial electron density $n_{\mathrm{e} 0}$, (b) observation weight $w$ (corresponding to the number of observations in each $\Delta L=0.15$ bin), (c) density power law index $\alpha$ (defined in equation (1)), and (d) effective field line scale length $L_{\alpha}=L R_{\mathrm{E}} / \sqrt{\alpha}$ versus $L$. In Figures $2 \mathrm{a}, 2 \mathrm{c}$, and $2 \mathrm{~d}$ the middle solid curve is the average value, while the upper and lower solid curves show the mean plus or minus one standard deviation. The large dashed curves in Figures $2 \mathrm{a}$ and $2 \mathrm{c}$ are a polynomial fit to the mean values.

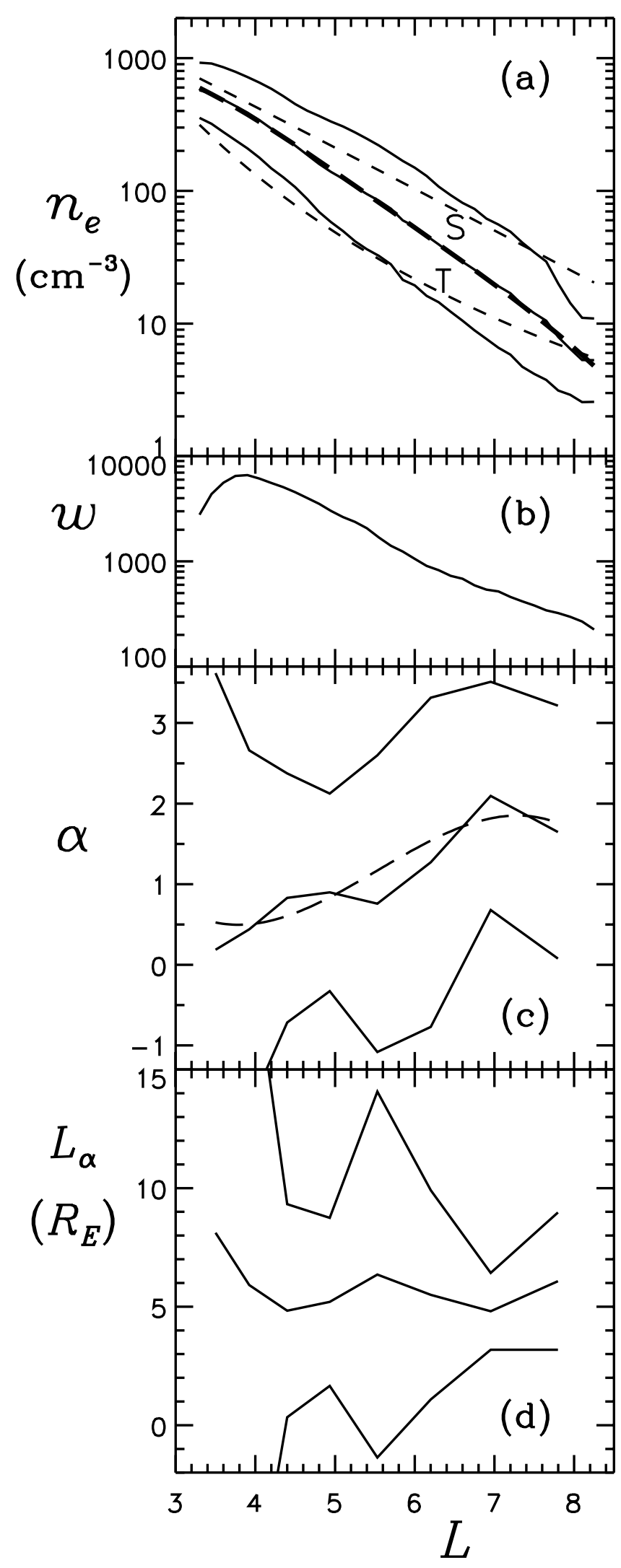


Table 1. Power Law Indices and Scale Lengths

\begin{tabular}{cccc}
\hline$L$ & $n_{\mathrm{e} 0}{ }^{\mathrm{a}}$ & $\alpha$ & $L_{\alpha}$ \\
\hline $3.5 \pm 0.2$ & $530 . \times \div 1.3$ & $0.2 \pm 3.4$ & $8.1 \pm 75$. \\
$3.9 \pm 0.2$ & $380 . \times \div 1.4$ & $0.4 \pm 2.2$ & $5.9 \pm 15$. \\
$4.4 \pm 0.3$ & $230 . \times \div 1.5$ & $0.8 \pm 1.5$ & $4.8 \pm 4.5$ \\
$4.9 \pm 0.3$ & $140 . \times \div 1.5$ & $0.9 \pm 1.2$ & $5.2 \pm 3.5$ \\
$5.5 \pm 0.3$ & $83 . \times \div 1.9$ & $0.8 \pm 1.8$ & $6.4 \pm 7.7$ \\
$6.2 \pm 0.4$ & $39 . \times \div 2.2$ & $1.3 \pm 2.0$ & $5.5 \pm 4.4$ \\
$7.0 \pm 0.4$ & $15 . \times \div 2.4$ & $2.1 \pm 1.4$ & $4.8 \pm 1.6$ \\
$7.8 \pm 0.4$ & $7.7 \times \div 2.4$ & $1.6 \pm 1.6$ & $6.1 \pm 2.9$ \\
\hline
\end{tabular}

a The notation " $\times \div$ " indicates that the uncertainty is a multiplicative factor.

(Since $n_{\mathrm{e}}$ rather than $n_{\mathrm{e} 0}$ is measured, equation (1) is used to convert each measurement of $n_{\mathrm{e}}$ to $n_{\mathrm{e} 0}$. Interpolation between the values of $\alpha$ from Table 1 , described below, is used to get the value of $\alpha$ for a particular measurement.) Figure $2 \mathrm{~b}$ shows the number of observations (observation weight) $w$ in each bin. The number of observations is the number of density data points measured and does not directly corresponding to time, for instance. Also, time periods for which there is no measurement of the upper hybrid resonance do not contribute to $w$. The middle solid curve in Figure $2 \mathrm{a}$ is the $\log$ average, while the upper and lower solid curves are found from the log average plus or minus one standard deviation (in log). The thick middle dashed curve overlying the middle solid curve is the polynomial fit to the log average which was used in the analysis,

$$
\begin{aligned}
\log _{10} n_{e 0-\bmod }= & 2.133+1.010 L-0.3656 L^{2}+0.0419 L^{3} \\
& -0.00182 L^{4}
\end{aligned}
$$

for $3.3 \leq L \leq 8.3$. However, an adequate fit to this data would appear to be

$$
\log _{10} n_{e 0-\bmod }=4.2-0.42 L \text {. }
$$

The upper thin dashed curve in Figure 2a marked " $\mathrm{S}$ " is the saturated plasmasphere density of Carpenter and Anderson [1992], while the lower thin dashed curve marked " $\mathrm{T}$ " is Carpenter and Anderson's plasmatrough density (varying roughly as $L^{-4.5}$ and with the density matching the $\log$ average value at $L=8$ ). Note that the average $n_{\mathrm{e}}$ is close to Carpenter and Anderson's saturated plasmapause density at $L=3.5$. At larger $L$ the average $n_{\mathrm{e}}$ is lower. One likely reason for this is that the spacecraft is sometimes in the plasmatrough, but we note also that Gallagher et al. [2000] found plasmaspheric densities lower than Carpenter and Anderson's saturated plasmasphere values at large $L$. Moving in the opposite direction from large $L$, the average $n_{\mathrm{e} 0}$ increases more steeply with respect to decreasing $L$ than Carpenter Anderson's plasmatrough density profile, again because plasmasphere densities make a relatively greater contribution to the average density at smaller $L$.

[14] We now pick eight $L$ values logarithmically spaced so as to fill the region between $L=3.5$ and 7.8. These $L$ values, $L_{\text {bin }}$, are used to bin the data by $L$, and their values are given in Table 1 along with the widths of the bins. For each $L_{\text {bin }}$ we examine the electron density versus the normalized radius $R /\left(L R_{\mathrm{E}}\right)$. For each observed value of $n_{\mathrm{e}}$ within the $L$ bin we first correct the density to account for differing $L_{\mathrm{obs}}$ value, where $L_{\mathrm{obs}}$ indicates the maximum radius on the field line connecting to the observation point. We do this by multiplying by $n_{e 0-\bmod }\left(L_{\mathrm{bin}}\right) / n_{e 0-\bmod }\left(L_{\mathrm{obs}}\right)$ using equation (2). (The careful reader will note here that we use $\alpha$ to get the model for $n_{\mathrm{e} 0}$ but also use our model for $n_{\mathrm{e} 0}$ to get $\alpha$. This is done by an iterative technique. In the first iteration we assume $\alpha=0$ so that $n_{\mathrm{e} 0}=n_{\mathrm{e}}$ for each measurement. After calculating the $\alpha$ values in Table 1 we subsequently use these values to correct $n_{\mathrm{e} 0}$ in further iterations.) Next, we choose 10 bins with $R /\left(L R_{\mathrm{E}}\right)$ ranging linearly from $3 / L_{\text {bin }}$ to 1.0 (recall that we are only using data with $R>3 R_{\mathrm{E}}$ ). We distribute the weight of each observation linearly between adjacent bin centers. That is, if a particular observation has $R /\left(L R_{\mathrm{E}}\right)$ halfway between two $R /\left(L R_{\mathrm{E}}\right)$ bins, that particular observation contributes an observation weight $w$ (number of observations) of 0.5 to each of these adjacent bins. In this manner we calculate a weighted log average of the density values in each bin. We throw out $R /\left(L R_{\mathrm{E}}\right)$ data points with $w<20$ to ensure that the results are statistically significant.

[15] Figure 3 shows $n_{\mathrm{e}}$ and observation weight $w$ (number of observations) versus $R /\left(L R_{\mathrm{E}}\right)$ for four of the $L_{\mathrm{bin}}$ values listed in Table 1 . In the plots of $n_{\mathrm{e}}$ the middle solid curve is the log average value, while the upper and lower solid curves result from the mean plus or minus one standard deviation (of the log). Note that there is a large variation in $n_{\mathrm{e}}$ from the upper to lower curves, a factor of 5 to 10 . Note also that the distribution of observations $(w)$ is not uniform with respect to $R /\left(L R_{\mathrm{E}}\right)$. In fact, if the magnetosphere were time stationary and north-south symmetric and the latitude of orbit apogee fixed, then the spacecraft would always cross a particular $L$ shell at the same value of $R /\left(L R_{\mathrm{E}}\right)$. However, owing to the seasonally dependent orientation of the dipole tilt and spacecraft meridian, variations in the magnetic field configuration caused by variations in the solar wind, and changes in the Polar orbit with time due to precession, the spacecraft does sample different values of $R /\left(L R_{\mathrm{E}}\right)$ at a particular $L$. We have examined the values of $R /\left(L R_{\mathrm{E}}\right)$ sampled with respect to various parameters. The strongest correlation appears to be with time and appears to result because of spacecraft precession. There was no significant correlation with $K p$ or Dst. Note also that the coverage of $R /\left(L R_{\mathrm{E}}\right)$ varies significantly with respect to $L$. The coverage at $L=5.5$ is very good compared with that at $L=3.5$ (owing in part to our restriction $R>3 / R E$ ).

[16] It can also be seen from Figure 3 that the density appears to be flatter with respect to $R /\left(L R_{\mathrm{E}}\right)$ at the low values of $L(L<7.0)$ than at the highest value $(L=7.0)$. This is shown more clearly in Figure 4 , which displays the log average density (solid curve) versus $R /\left(L R_{\mathrm{E}}\right)$ for each of the $L=L_{\text {bin }}$ values listed in Table 1 . The vertical range is a factor of 6 in each frame so that the relative variation in $n_{\mathrm{e}}$ can be compared. Both the horizontal and vertical axes are plotted using a log scale. The dashed lines in each panel are a linear fit to $\log _{10} n_{\mathrm{e}}$ versus $\log _{10} R /\left(L R_{\mathrm{E}}\right)$, and these fits yield the values of $n_{\mathrm{e} 0}$ and $\alpha$ in equation (1) which are listed in Table 1. These $\alpha$ values are plotted in Figure $2 \mathrm{c}$ as the middle solid curve. The upper and lower solid curves 


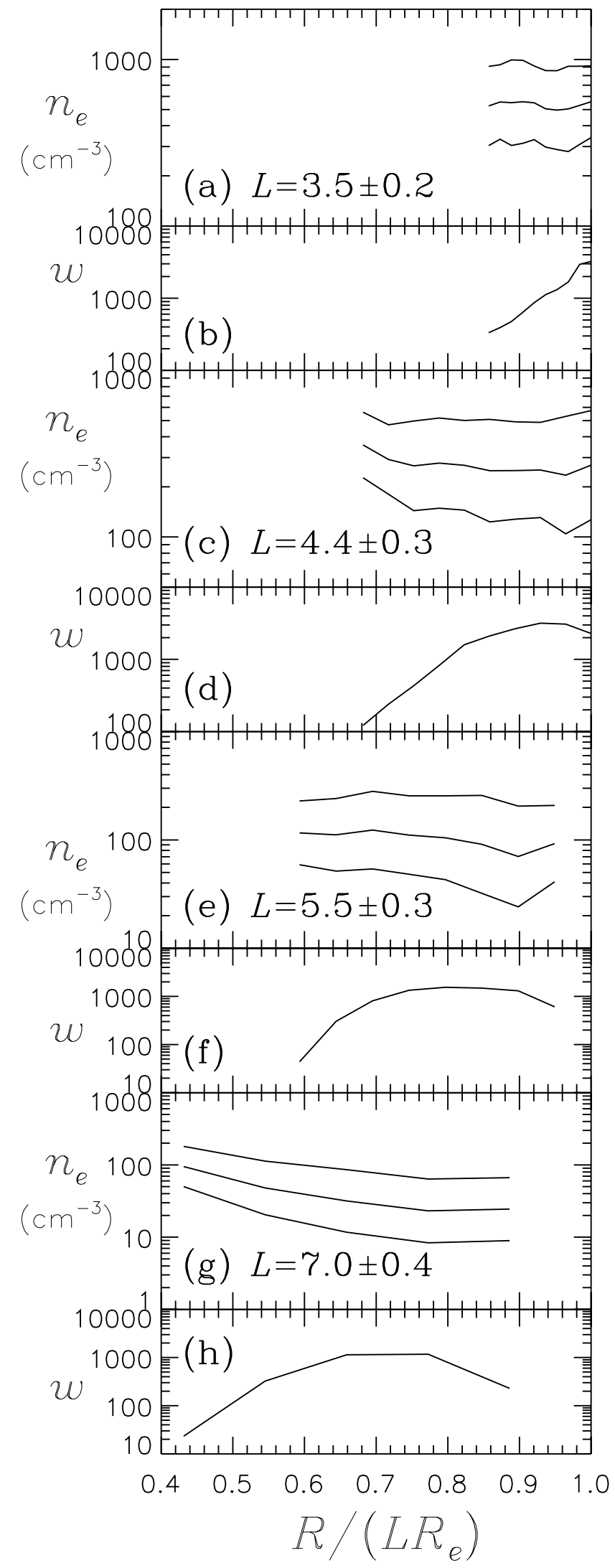

Figure 3. Electron density $n_{\mathrm{e}}((\mathrm{a}),(\mathrm{c}),(\mathrm{e})$, and $(\mathrm{g}))$ and observation weight $w((\mathrm{~b}),(\mathrm{d}),(\mathrm{f})$, and $(\mathrm{h}))$ versus $R /\left(L R_{\mathrm{E}}\right)$ for $L=L_{\text {bin }}=3.5 \pm 0.2$ (Figures $3 \mathrm{a}$ and $3 \mathrm{~b}$ ), $L_{\text {bin }}=4.4 \pm 0.3$ (Figures $3 \mathrm{c}$ and $3 \mathrm{~d}$ ), $L_{\text {bin }}=5.5 \pm 0.3$ (Figures $3 \mathrm{e}$ and $3 \mathrm{f}$ ), and $L_{\text {bin }}=7.0 \pm 0.4$ (Figures $3 \mathrm{~g}$ and $3 \mathrm{~h}$ ).

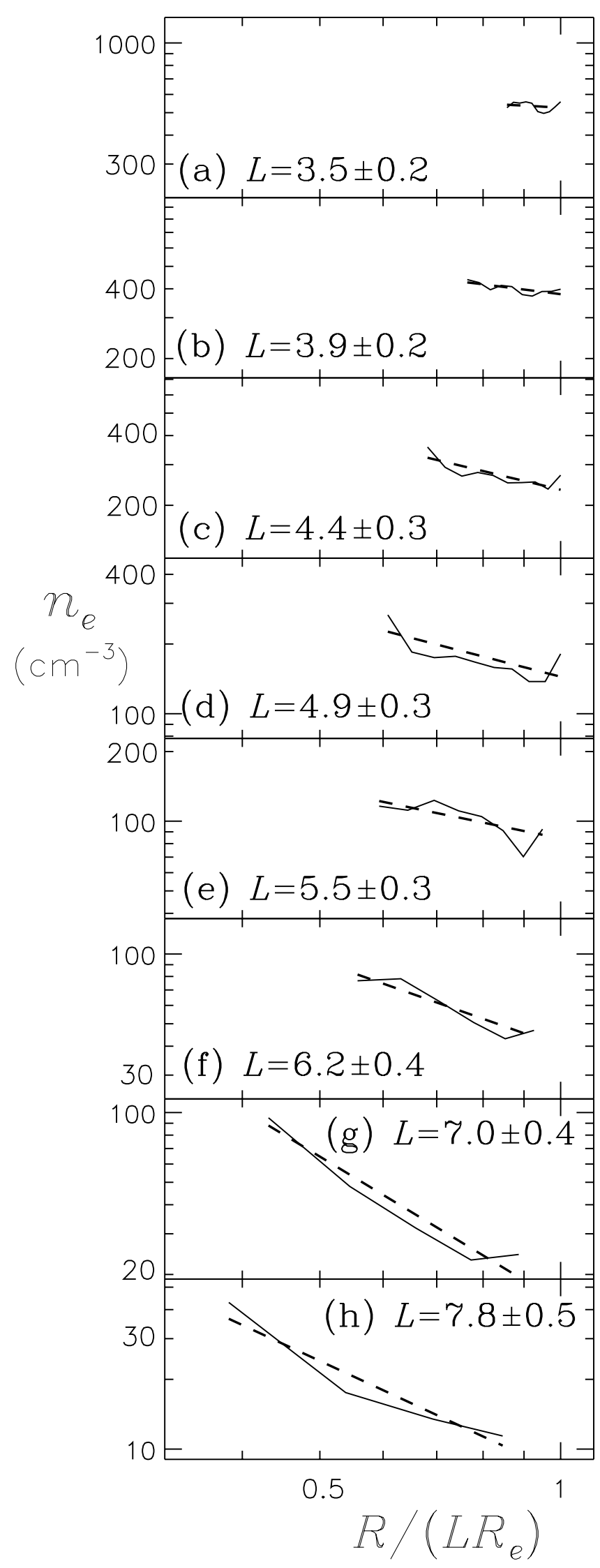

Figure 4. Plot of $n_{\mathrm{e}}$ versus $R /\left(L R_{\mathrm{E}}\right)$ for each of the $L=L_{\text {bin }}$ values listed in Table 1. The solid (dashed) curve is the binned log average (linear fit). 
represent the uncertainty of $\alpha$ from the linear fit [Press et al., 1997], and the dashed curved line is a crude polynomial fit

$$
\begin{aligned}
\alpha_{\mathrm{fit}} & =1.66+0.565 x-0.465 x^{2}+0.0630 x^{3}, \\
x & =8-L,
\end{aligned}
$$

for $3.5 \leq L \leq 7.8$.

[17] Larger $\alpha$ corresponds to a steeper increase in $n_{\mathrm{e}}$ with respect to decreasing $R /\left(L R_{\mathrm{E}}\right)$ (equation (1)). As is clear from Figure 2c, $\alpha$ is larger at larger $L$. This result is quite consistent with the results of Goldstein et al. [2001], who found $\alpha=0.37 \pm 0.8$ for their plasmaspheric data set $\left(n_{\mathrm{e}} \geq\right.$ $100 \mathrm{~cm}^{-3}$ ), but $\alpha=1.7 \pm 1.1$ for their plasmatrough data set $\left(n_{\mathrm{e}}<100 \mathrm{~cm}^{-3}\right)$. In fact, Figure $2 \mathrm{c}$ appears to give evidence of two regions with different values of $\alpha$. Note from Figure $2 \mathrm{c}$ that the average $\alpha$ is relatively flat with respect to $L$ in the region $L \leq 5.5$ (which usually corresponds to the plasmasphere) and in the region $L \geq 7$ (which usually corresponds to the plasmatrough). There appears to be a transition region with a mix of the two types for $5.5 \leq L \leq 7$. Around the average value there is a large uncertainty in $\alpha$ (Table 1 and Figure 2c). Our uncertainty values for $\alpha$ are probably overestimates since the uncertainty in $\alpha$ for particular field lines may be less than the uncertainty found from the entire distribution of densities. In fact, Goldstein et al. [2001], who matched pairs of density values to infer $\alpha$, found lower uncertainties $(\Delta \alpha \sim 1)$ and they considered their uncertainties to be overestimates.

[18] The variation of density along the field line can also be described in terms of a scale length. We define the scale length $\left.L_{\alpha} \equiv \sqrt{2 n_{\mathrm{e} 0} /\left(d^{2} n_{\mathrm{e}} / d l^{2}\right)}\right|_{l=0}$, where $l$ is the length along the field line defined to be zero at the magnetic equator. Assuming $d n_{\mathrm{e}} /\left.d l\right|_{l=0}=0$ at the magnetic equator (implied by equation (1)), $L_{\alpha}$ is the distance along the field line from the equator at which the density is twice the equatorial value. In a dipole field, $L_{\alpha}=\left(L R_{\mathrm{E}}\right) / \sqrt{\alpha}$, and based on the values of $\alpha$ already calculated, values of this quantity are listed in Table 1 and plotted in Figure 2d. From Figure $2 \mathrm{~d}$ we see that $L_{\alpha}$ is relatively constant. Averaging the last six values of $L_{\alpha}$ listed in Table 1 (for which the uncertainty is relatively less), we find $L_{\alpha}=5.5 \pm$ 4.1 for $4.4 \leq L \leq 7.8$ (where the error noted is an overestimate).

[19] Figure 5 is like Figure 2 except that the data is separated into four groups according to MLT. The thin solid curve in Figure 5 corresponds to the midnight sector MLT $=21-03$, the thin dashed curve corresponds to dawn MLT $=03-09$, the thick solid curve corresponds to noon MLT $=09-15$, and the thick dashed curve corresponds to dusk MLT $=15-21$. It is clear that the density is depleted in the dawn sector (MLT $=03-09$ ) and that the radial dependence of the density is steeper there ( $\alpha$ larger). These results are consistent with erosion from MLT $=0-6$ (leading to low density with steep radial dependence at dawn) and refilling for MLT $=6-18$ (leading to milder radial dependence at noon). Alternately, one might say that there is a greater probability of plasmatrough density in the dawn local time sector (the plasmapause is typically at its lowest $L$ shell near dawn).

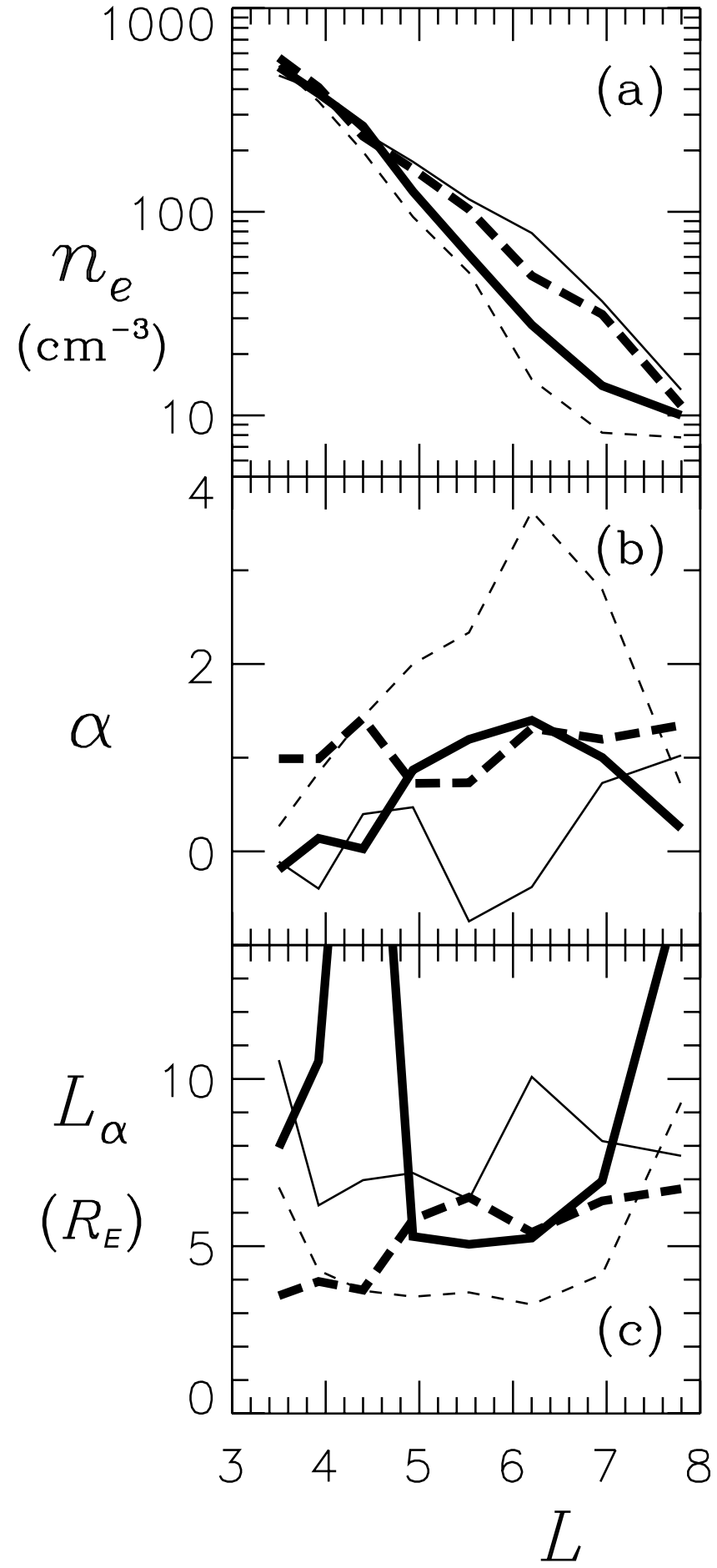

Figure 5. Plot similar to Figure 2 with (a), (b) and (c) corresponding to Figure 2a, Figure 2c, and Figure 2d, respectively. Here the data is plotted for different MLT regions, 21-03 (thin solid curve), 03-09 (thin dashed curve), 09-15 (thick solid curve), and 15-21 (thick dashed curve).

[20] Similarly, in Figure 6 we have separated the data into two groups, geomagnetically quiet times with $\langle K p\rangle<1.2$ (solid curve) and active times with $\langle K p\rangle>2$ (dashed curve). Here $\langle K p\rangle$ represents a value of $K p$ averaged over the preceding time $t$ using the weighting factor $\exp -\left(t / t_{0}\right)$, 


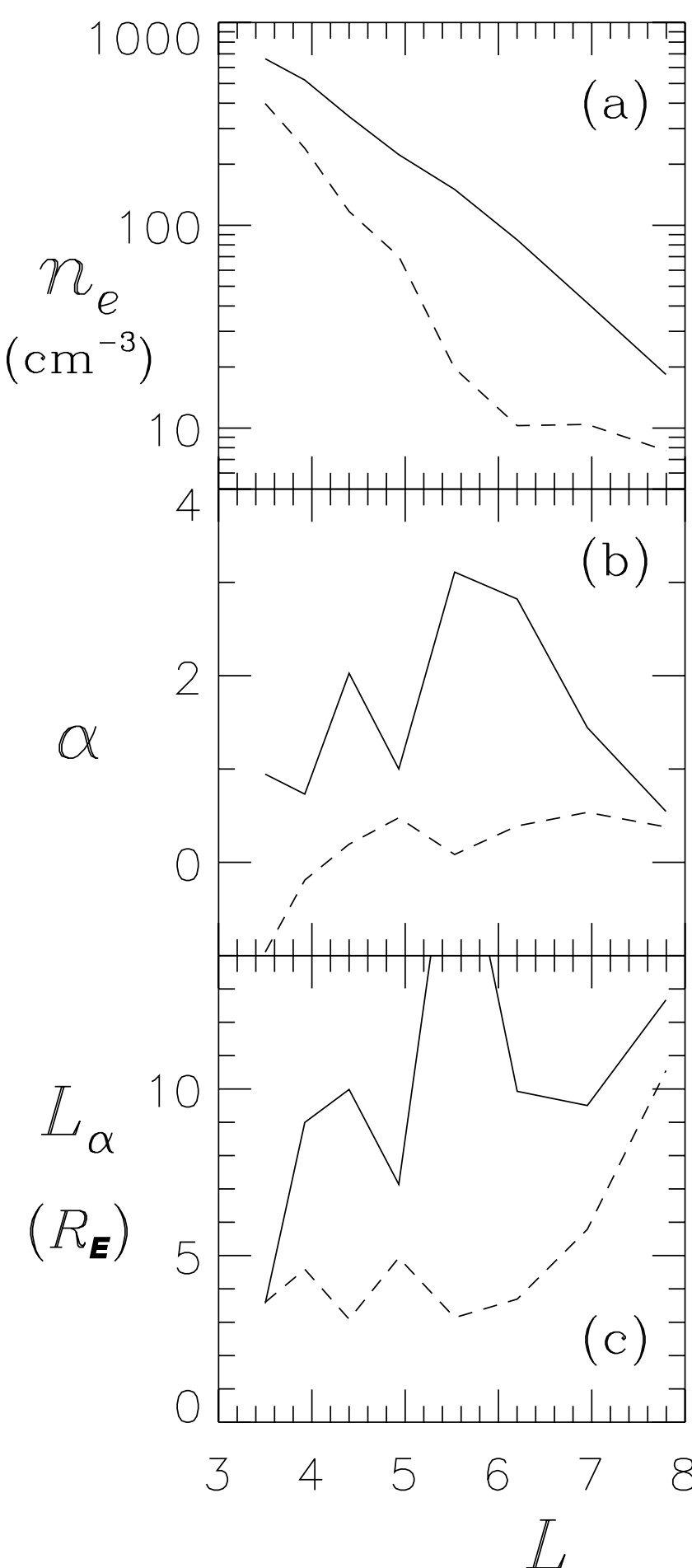

Figure 6. Like Figure 5 except that the data is plotted for different average values of $K p,\langle K p\rangle<1.2$ (solid curve) and $\langle K p\rangle>2$ (dashed curve) (the averaging procedure is described in the text).

where $t_{0}=1.5$ days (following a similar procedure to that of Gallagher et al. [1988]). Figure 6 shows that during more active times $(\langle K p\rangle>2)$, the density is depleted and the radial dependence is steeper. Again, the steeper radial dependence may be related to a greater proportion of plasmatrough density. In both Figure 2 and Figure 6 the density values below $10 \mathrm{~cm}^{-3}$ represent an upper limit, as indicated in the last sentence of section 2 .

\section{Discussion and Conclusions}

[21] Using measurements by the Polar spacecraft Plasma Wave Instrument (PWI), we have calculated the average magnetospheric electron density in the region $3.5<L<7.8$ as a function of $R /\left(L R_{\mathrm{E}}\right)$ (averaging over all quantities except $L$ and $\left.R /\left(L R_{\mathrm{E}}\right)\right)$. One of the interesting results of this study is that the power law form seems to do an adequate job of describing at least the average density variation along field lines (Figure 4). Of course it must be remembered that our study is limited to the region relatively close to the equator. Equations (2) or (3) with equation (4) or interpolation between the $\alpha$ values in Table 1 constitutes a crude electron density model for $3.5 \leq L \leq 7.8$. While the lack of a plasmapause in equation (2) makes it unlikely that the real plasma profile has at any time the dependence described in that equation, it still can be used as a constraint on global density models and for situations where a model is desired with an average density (averaging over plasmasphere and plasmatrough conditions). Caution should be exercised where the model values for $n_{\mathrm{e} 0}$ drop below about 10 $\mathrm{cm}^{-3}$, since the upper hybrid noise band is sometimes undetectable at low densities (Figure 1).

[22] The values of $\alpha$ listed in Table 1 give information about the field line variation of $n_{\mathrm{e}}$ (see equation (1)). On the basis of Figure 2c, $\alpha$ appears to have on average a value $0.2-0.9$ in the plasmasphere (inner $L$ region, $3.5 \leq L \leq 5.5$ ) and a value $1.6-2.1$ in the plasmatrough (outer $L$ region, $L \geq 7$ ). For instance, $\alpha=0.8 \pm 1.2$ at $L=4.4$ and $\alpha=2.1 \pm$ 1.4 at $L=7$ (where the errors noted are overestimates). Our results are consistent with those of Goldstein et al. [2001], who found on average $\alpha \sim 0.4$ in the plasmasphere and $\alpha \sim$ 1.7 in the plasmatrough. As discussed by [Goldstein et al., 2001], these values indicate that the field line dependence of $n_{\mathrm{e}}$ is roughly consistent with diffusive equilibrium in the plasmasphere $(\alpha \sim 0)$ but that the steepness of density in the plasmatrough is in between that of diffusive equilibrium and kinetic models ( $\alpha \sim 3-4)$. Of course, it should be remembered that the uncertainty in our $\alpha$ values is quite large (Table 1 or Figure $2 \mathrm{c}$ ). These uncertainties are probably overestimates, as discussed in the second to last paragraph of section 3. Another important thing to note is that the data coverage of $R /\left(L R_{\mathrm{E}}\right)$ varies as a function of $L$ (Figure 4$)$, so that from the point of view of $R /\left(L R_{\mathrm{E}}\right)$ we are not sampling exactly the same section of the field line at different $L$ values.

[23] We have also separated the data according to MLT; in the dawn sector the density is depleted and the radial dependence is steeper (larger $\alpha$ ) than for other MLT sectors (Figure 5). A similar result holds when the averaged $K p$ value is large $(>2)$ (Figure 6). It should be kept in mind that these conditions correspond to a higher proportion of plasmatrough data.

[24] The field line studied by Reinisch et al. [2001], $L=3$, is outside the range of our study. Nevertheless, interpreting their results in terms of equation (1) as discussed in the fourth paragraph of section 1 , the value $\alpha \sim 0.75$ implied by their results in the vicinity of the equator is consistent with our values for $\alpha$. While their dependence at $40^{\circ}, \alpha \sim 3$, is 
steeper than our average value for $\alpha$, this location is also getting rather close to the Earth $(R=1.76)$ and is never sampled by Polar. (Actually, if we keep the data for $R>2$ rather than $R>3$, we find that $\alpha$ increases for $L<3$, but we have thrown out this data for the reasons discussed in the third paragraph of section 2.) Our results on field line variation of $n_{\mathrm{e}}$ are neatly summarized by the result that the scale length $\left.L_{\alpha} \equiv \sqrt{2 n_{e 0} /\left(d^{2} n_{e} / d l^{2}\right)}\right|_{l=0}=5.5 \pm 4.1 R_{\mathrm{E}}$ over the fairly large region $4.4 \leq L \leq 7.8$ (where the error noted is an overestimate).

[25] Acknowledgments. We are grateful to Iowa PWI team, including D. Gurnett and J. Pickett, for supplying the upper hybrid resonance data (supported by NASA contract NAS5-30371 and grant NAG5-7943), the Polar spacecraft science data team for supplying spacecraft ephemeris data, and NSSDC for making the IMP-8 (GSFC and LANL) and WIND (courtesy of R. P. Lepping and K. W. Ogilvie) solar wind data, Dst (Kyoto University), and $K p$ (NOAA) data available on OMNIWeb. We thank Dennis Gallagher for providing the averaged $K p$ data. Work at Dartmouth was supported by NASA grants NAG5-7442 and NSF grant ATM9911975. Work at the University of Iowa was supported by subcontract 14280500 to MSFC/NASA project NAG8-1153.

[26] Lou-Chuang Lee and Chin S. Lin thank Dennis L. Gallagher and Frederick W. Menk for their assistance in evaluating this paper.

\section{References}

Angerami, J. J., and D. L. Carpenter, Whistler studies of the plasmapause in the magnetosphere, 2, Electron density and total tube electron content near the knee in magnetospheric ionization, J. Geophys. Res., 71, 711, 1966.

Carpenter, D. L., and R. R. Anderson, An ISEE/whistler model of equatorial electron density in the magnetosphere, J. Geophys. Res., 97, 1097, 1992.

Chan, A. A., M. Xia, and L. Chen, Anisotropic Alfvén-ballooning modes in Earth's magnetosphere, J. Geophys. Res., 99, 17,351, 1994.

Cummings, W. D., R. J. O’Sullivan, and P. J. Coleman Jr., Standing Alfvén waves in the magnetosphere, J. Geophys. Res., 74, 778, 1969.

Denton, R. E., M. R. Lessard, R. Anderson, E. G. Miftakhova, and J. W. Hughes, Determining the mass density along magnetic field lines from toroidal eigenfrequencies: Polynomial expansion applied to CRRES data, J. Geophys. Res., 106, 29,915, 2001.

Engebretson, M. J., L. J. Zanetti, and T. A. Potemra, Harmonically structured ULF pulsations observed by the AMPTE CCE magnetic field experiment, Geophys. Res. Lett., 13, 905, 1986.

Gallagher, D. L., P. D. Craven, and R. H. Comfort, An empirical model of the Earth's plasmasphere, Adv. Space Res., 8, 15, 1988.
Gallagher, D. L., P. D. Craven, and R. H. Comfort, Global core plasma model, J. Geophys. Res., 105, 18,819, 2000.

Goldstein, J., R. E. Denton, M. K. Hudson, E. G. Miftakhova, S. L. Young, J. D. Menietti, and D. L. Gallagher, Latitudinal density dependence of magnetic field lines inferred from Polar plasma wave data, J. Geophys., $106,6195,2001$.

Gurnett, D. A., et al., The Polar Plasma Wave Instrument, Space Sci. Rev, 71, 583, 1995.

Kolesnikova, E., C. Beghin, R. Grard, and C. P. Escoubet, The electrical stability of the electric field antennas in the plasmasphere, J. Atmos. Terr. Phys., 63, 1217, 2001.

Langel, R. A., International geophysical reference field, 1991 revision, J. Geomag. Geoelectr., 43, 1007, 1991.

Lemaire, J. F., and K. I. Gringauz, The Earth's Plasmasphere, pp. 222-249, Cambridge Univ. Press, New York, 1998.

Menk, F. W., D. Orr, M. A. Clilverd, A. J. Smith, C. L. Waters, D. K. Milling, and B. J. Fraser, Monitoring spatial and temporal variations in the dayside plasmasphere using geomagnetic field line resonances, J. Geophys. Res., 104, 19,955, 1999.

Press, W. H., S. A. Teukolsky, W. T. Vetterling, and B. P. Flannery, Numerical Recipes in Fortran 77: The Art of Scientific Computing, Fortran Numerical Recipes, vol. 1, Cambridge Univ. Press, New York, 1997.

Price, I. A., C. L. Waters, F. W. Menk, G. J. Bailey, and B. J. Fraser, A technique to investigate plasma mass density in the topside ionosphere using ULF waves, J. Geophys. Res., 104, 12,723, 1999.

Reinisch, B. W., X. Huang, P. Song, G. S. Sales, S. F. Fung, J. L. Green, D. L. Gallagher, and V. M. Vasyliunas, Plasma density distribution along the magnetospheric field: RPI observations from IMAGE, Geophys. Res. Lett., 28, 4521, 2001.

Takahashi, K., and R. L. McPherron, Harmonic structure of Pc 3-4 pulsations, J. Geophys. Res., 87, 1504, 1982.

Tsyganenko, N. A., A magnetospheric magnetic field model with a warped tail current sheet, Planet. Space Sci., 37, 5, 1989.

Tsyganenko, N. A., Modeling the Earth's magnetospheric magnetic field confined within a realistic magnetopause, J. Geophys. Res., 100, 5599, 1995.

Young, E. R., D. G. Torr, P. Richards, and A. F. Nagy, A computer simulation of the midlatitude plasmasphere and ionosphere, Planet. Space Sci., $28,881,1980$.

R. E. Denton, Department of Physics and Astronomy, 6127 Wilder Laboratory, Dartmouth College, Hanover, NH 03755, USA. (Richard. Denton@dartmouth.edu)

J. Goldstein, Department of Physics and Astronomy, MS-108, Rice University, 6100 Main Street, Houston, TX 77005-1892, USA. (jerru@ hydra.rice.edu)

J. D. Menietti, Department of Physics and Astronomy, University of Iowa, Iowa City, IA 52242, USA. (jdm@space.physics.uiowa.edu)

S. L. Young, AFRL/VSBXR, 29 Randolph Road, Hanscom AFB, MA 01731-3010, USA. (Shawn.Young@Hanscom.AF.mil) 